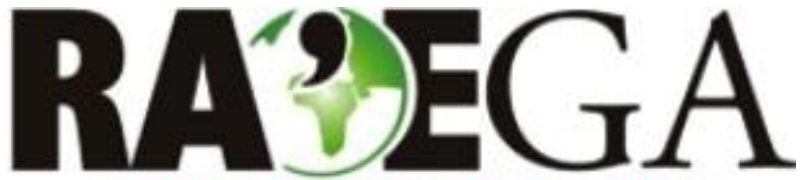

O ESPAÇO GEOGRÁFICO EM ANÁLISE

\title{
IDENTIFICAÇÃO DE ÁREAS COM PERDA DE SOLO ACIMA DO TOLERÁVEL USANDO NDVI PARA O CÁLCULO DO FATOR C DA USLE
}

\section{IDENTIFICATION OF AREAS WITH SOIL LOSS ABOVE THE TOLERABLE USING NDVI FOR CALCULATING THE C FACTOR OF USLE}

\author{
Darllan Collins da Cunha e Silva ${ }^{1}$, José Luiz Albuquerque Filho ${ }^{2}$, Jomil Costa Abreu Sales ${ }^{3}$, Roberto \\ Wagner Lourenço ${ }^{4}$
}

\section{RESUMO}

Um dos principais problemas relacionados à conservação dos solos das bacias hidrográficas é a erosão hídrica do solo com o consequente transporte de partículas do solo aos corpos hídricos, especialmente em regiões com presença de atividades agrícolas. Portanto, o presente estudo teve como objetivo demonstrar a aplicação de uma metodologia para calcular o fator C por meio do Normalized Difference Vegetation Index (NDVI) utilizando técnicas de geoprocessamento e modelagem matemática para quantificar a perda de solo na Bacia Hidrográfica do Rio Una através da utilização da Equação Universal de Perda de Solo (USLE) e, por fim, avaliar as diferentes perdas de solo encontradas de acordo com os diferentes usos e ocupações do solo. Os resultados mostraram um baixo potencial de perda de solo, que é consequência da associação de diferentes fatores, em especial, devido a cobertura vegetal que corresponde a $41 \%$ da extensão territorial da bacia hidrográfica e foi responsável por manter as taxas de erosão menores que 10 t.ha-1.ano- 1 em 93,4\% da área de estudo, enquanto que, as taxas de erosão superiores a 10 t.ha1.ano-1 encontram-se em locais com atividades agrícolas e altas declividades. Os métodos aplicados demonstraram ser eficientes e de grande contribuição para estudos de bacias hidrográficas com vistas ao planejamento, manejo e preservação do solo.

PALAVRAS-CHAVE: Geoprocessamento; USLE; Bacia Hidrográfica; Erosão.

\section{ABSTRACT}

One of the main problems related to the conservation of the watershed soils is the hydric erosion of the soil, leading to particles transportation to water bodies as consequence, especially in those regions with agricultural activities. Therefore, this study main purpose was to demonstrate the factor c calculation methodology application, through the Normalized Difference Vegetation Index (NDVI) using geoprocessing techniques and mathematical modeling to quantify the soil loss in the Una River Basin, through the Universal Soil Loss Equation (USLE), it was possible to evaluate the different soil losses found according to thedifferent land uses and occupations. The results showed a low soil loss potential, which is a result of the association of different factors, in special, vegetation cover, which corresponds to $41 \%$ of the watershed area, and was also responsible for maintaining erosion rates lower than 10 t.ha-1.year-1 in 93,4\% from the study area, while erosion rates higher than $10 \mathrm{t}$ ha-1.year-1 werelocated within areas with agricultural activities and high steepness. The applied methods have proven to be efficient and highly contribute to river basins studies regarding the planning, management and soil conservation.

KEY-WORDS: Geoprocessing; USLE; Watershed; Erosion.

\footnotetext{
1 Universidade Estadual Paulista "Júlio de Mesquita Filho", UNESP, Sorocaba/SP, e-mail: darllanamb@yahoo.com.br

${ }^{2}$ Centro de Tecnologias Geoambientais do Instituto de Pesquisas Tecnológicas - IPT, São Paulo/SP,e-mail: albuzelu@ipt.br

3 Universidade Estadual Paulista "Júlio de Mesquita Filho", UNESP, Sorocaba/SP, e-mail: jomilc@gmail.com

${ }^{4}$ Universidade Estadual Paulista "Júlio de Mesquita Filho", UNESP, Sorocaba/SP, e-mail: robertow@sorocaba.unesp.br
} 


\section{IDENTIFICAÇÃO DE ÁREAS COM PERDA DE SOLO ACIMA DO TOLERÁVEL USANDO NDVI PARA O CÁLCULO DO FATOR C DA USLE}

\section{INTRODUÇÃO}

A erosão pluvial é um processo natural que ocorre ao longo do tempo geológico modelando a superfície da Terra. Entretanto, a retirada e substituição de extensas áreas com cobertura vegetal para a implantação de culturas agrícolas e processos de urbanização resultam em uma maior exposição do solo aos fatores intempéricos, permitindo, desse modo, que uma grande quantidade de partículas do solo seja desagregada e carreada pelo impacto da chuva e por enxurradas aos cursos de água provocando até assoreamento desses corpos de águas (BERTONI; LOMBARDI NETO, 1999). Além disso, estas partículas do solo desagregadas e transportadas pelo escoamento superficial aumentam a possibilidade de ocorrência de cheias, podendo gerar uma poluição hídrica devido aos elementos químicos carreados, tais como fertilizantes e pesticidas (FUGIHARA, 2002).

A necessidade de compreender os processos erosivos e suas consequências para gerir de forma sustentável as atividades antrópicas possibilitou a geração de diferentes modelos matemáticos a partir da década de 1940. Wischmeier e Smith (1978) foram os responsáveis pela criação da "Universal Soil Loss Equation" (USLE), modelo que estima a perda de solo por erosão hídrica. Posteriormente, a USLE foi adaptada e assim criada a "Revised Universal Soil Loss Equation" (RUSLE) que é um modelo de base empírica que calcula a erosão do solo através de valores determinados por índices que representam os principais fatores, tais como chuva, solo, topografia e uso do solo (RENARD et al., 1997). De acordo com Galdino e Weill (2011) as estruturas das equações da RUSLE e USLE são idênticas, o que as diferencia é a maneira como são determinados os fatores da equação.

No Brasil, a RUSLE não é sistematicamente usada para identificar os pontos críticos de perda de solo, tão pouco como ferramenta de planejamento e gestão, certamente pela falta de dados, o que dificulta a alimentação do modelo. Dentre os fatores da RUSLE, o fator C é aquele que trata da gestão do uso do solo praticado em uma determinada área e o principal fator que pode ser planejado a fim de selecionar as áreas mais aptas a determinadas atividades antrópicas, pois representa o efeito da cobertura e manejo do solo na redução da erosão hídrica (BERTONI; LOMBARDI NETO, 2012).

Segundo Medeiros et al. (2010), dentre os fatores que compõem a RUSLE, o fator C é o que apresenta maiores incertezas, uma vez que, encontraram em seus estudos, uma grande variabilidade em comparação aos outros fatores para uma mesma área de estudo, com valores máximos e mínimos variando 74 vezes, enquanto que, outros fatores como o de erodibilidade e topográfico variaram até 2 e 5 vezes respectivamente. Bertoni e Lombardi Neto et al. (2012) atribui a essa incerteza, a dinâmica do uso do solo agrícola e a necessidade de determinar com precisão a cultura e o manejo praticado. Amorim et al. (2010) corroborando com essas afirmações, concluíram em seus estudos que os maiores desvios encontrados nas estimativas da RUSLE são explicados, em parte, pela falta de dados para a determinação precisa deste fator no Brasil.

Aliado aos modelos matemáticos da RUSLE e USLE, o geoprocessamento é uma importante ferramenta para auxiliar na quantificação e qualificação da perda de solo por erosão hídrica, pois é capaz de realizar operações analíticas, sobreposição e cruzamento de informações, permitindo a análise e modelagem dos fatores dos propostos em equações que avaliam a perda de solo (COSTA, 2005; MINGOTI, 2009).

Diante do exposto, o presente trabalho teve como objetivo demonstrar a aplicação de uma metodologia para calcular o fator $\mathrm{C}$ por meio do Normalized Difference Vegetation Index (NDVI) utilizando técnicas de geoprocessamento e modelagem matemática para quantificar a perda de solo na Bacia Hidrográfica do Rio Una através da utilização da Equação Universal de Perda de Solo (USLE) e, por fim, avaliar as diferentes perdas de solo encontradas de acordo com os diversos tipos de usos e ocupações destes. Sua relevância justifica-se na medida em 


\section{IDENTIFICAÇÃO DE ÁREAS COM PERDA DE SOLO ACIMA DO TOLERÁVEL USANDO NDVI PARA O CÁLCULO DO FATOR C DA USLE}

que auxilia no planejamento do uso, manejo e conservação do solo.

\section{METODOLOGIA}

\section{CARACTERIZAÇÃO DA ÁREA DE ESTUDO}

A área de estudo, denominada Bacia Hidrográfica do Rio Una, faz parte da décima Unidade de Gerenciamento de Recursos Hídricos do Sorocaba e Médio Tietê (UGHRI 10) e está localizada no interior do Estado de São Paulo a aproximadamente $75 \mathrm{~km}$ da capital do Estado de São Paulo, no Município de Ibiúna (Figura 1), conforme carta de São Roque SF-23-Y-C-V-2 do Instituto Brasileiro de Geografia e Estatística (IBGE, 1979). O município de Ibiúna está inserido na Região Metropolitana de Sorocaba (RMS) e possui uma população de 73.309 habitantes com
$55,7 \%$ dos habitantes residentes na zona rural, segundo dados publicados pela Fundação Sistema Estadual de Análise de Dados referentes ao ano de 2014 (SEADE, 2015).

O Rio Una juntamente com os Rios Sorocabuçu e o Sorocamirim desembocam no reservatório de Itupararanga, o qual possui grande importância regional, sendo o principal manancial de captação de água para o município de Sorocaba e região, além de irrigar centenas de propriedades agrícolas nos arredores. O clima predominante na área de estudo é do tipo $\mathrm{Cwb}$, isto é, com verão chuvoso e inverno com estiagem, segundo a classificação de Köeppen, enquanto que, a vegetação original é a ombrófila densa montana (VELOSO et al., 1991; SALLES, 2008; SALES, 2015).

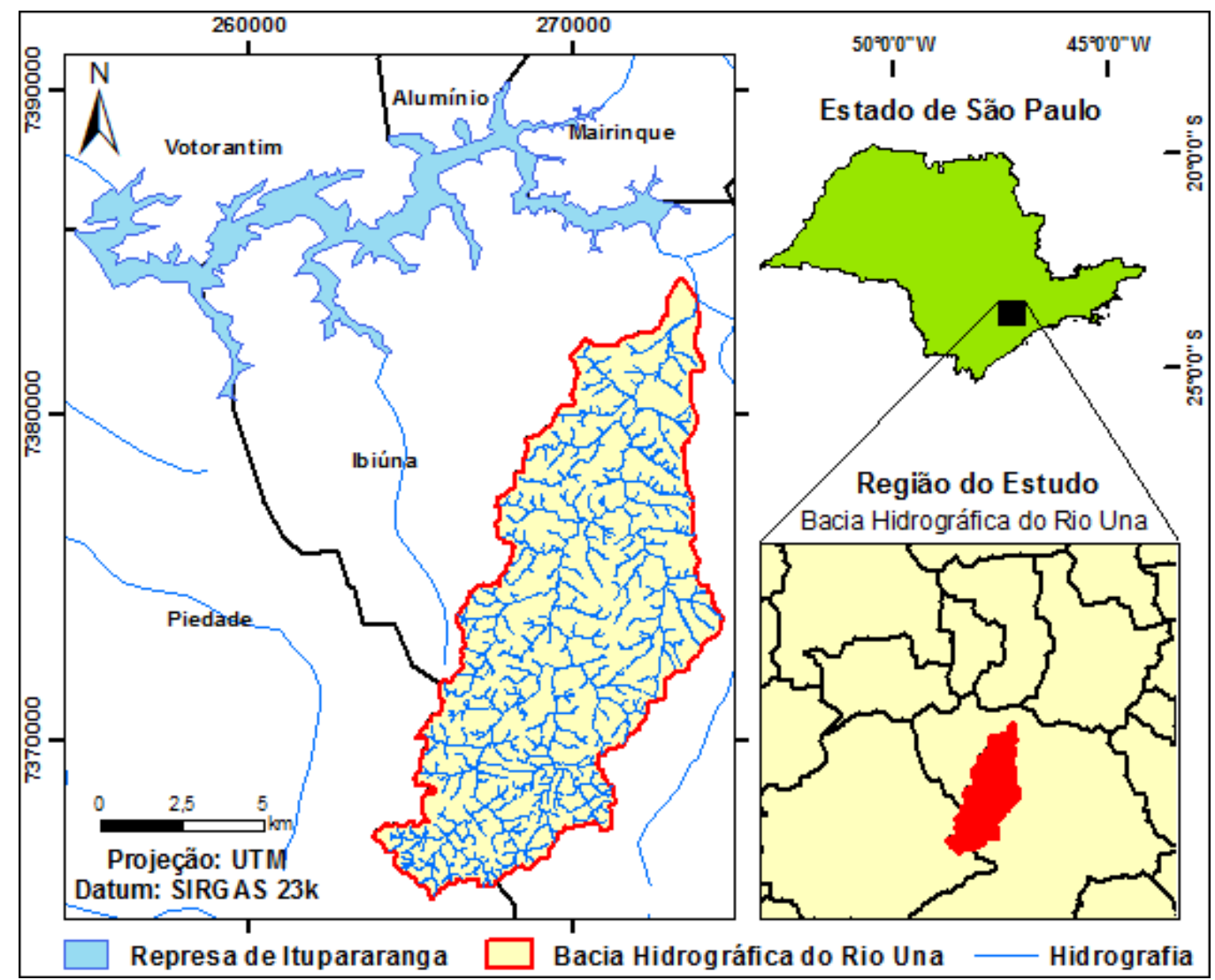

Figura 01 - Localização da Bacia Hidrográfica do Rio Una

ELABORAÇÃO DO MAPA DE USO E OCUPAÇÃO DO SOLO

Para o mapeamento do uso do solo e cobertura vegetal foram utilizadas imagens de satélite do sensor orbital SPOT-5 do ano de 2013.
A partir dessas imagens foram realizadas composições coloridas (RGB) e de falsa-cor através da utilização das bandas espectrais do infravermelho próximo e médio para a extração das feições relacionadas ao uso do solo e 


\section{IDENTIFICAÇÃO DE ÁREAS COM PERDA DE SOLO ACIMA DO TOLERÁVEL USANDO NDVI PARA O CÁLCULO DO FATOR C DA USLE}

cobertura vegetal. Para a análise dessas composições foi empregado o método de classificação supervisionada multivariada de Máxima Verossimilhança do software ArcGIS 10.1 .

As classes do mapa de uso do solo e cobertura vegetal da área de estudo foram divididas em: Pastagens; Área Florestal (Matas); Lagos, Açudes e Represas; Edificações Antrópicas Presentes em Áreas Urbanas; Edificações Antrópicas Presentes em Áreas Rurais; Campos Sujos; Agricultura com Culturas Perenes e Agricultura com Culturas Temporárias, de acordo com os níveis I e II presentes no Manual Técnico de Uso da Terra (IBGE, 2013), com exceção das áreas com edificações antrópicas que foram divididas em Edificações Antrópicas Presentes em Áreas Urbanas e Edificações Antrópicas Presentes em Áreas Rurais de acordo com a classificação dos setores censitários presentes no Censo Demográfico de 2010 (IBGE, 2012). Além disso, foram realizadas visitas a campo para validar a classificação do uso do solo e cobertura vegetal.

\section{ELABORAÇÃO DO MAPA DE PERDA DE SOLO}

Para a construção do mapa de perdas de solos foi utilizado a metodologia proposta por Wischmeier e Smith (1978), cujo cálculo da perda de solo por erosão hídrica é obtido através da Equação Universal de Perda de Solo (USLE). Esta metodologia permite estimar a perda de solo média anual em diferentes condições de uso e manejo do solo, variação climática, relevo e utilização de práticas conservacionistas, de modo a determinar áreas que apresentam maiores ou menores perdas de solos. A USLE é expressa pela Equação (1):

$$
A=R \cdot K \cdot L S \cdot C \cdot P
$$

Onde:

A - perda de solo por unidade de área no tempo (t.ha- ${ }^{-1}$ );

$\mathrm{R}$ - fator erosividade da chuva (MJ.mm.h $\mathrm{h}^{-1} \cdot \mathrm{ha}^{-1}$ );

$\mathrm{K}$ - fator de erodibilidade do solo (t.ha.h/ ha.MJ.mm);

LS - fator topográfico englobando a declividade e comprimento de rampa (adimensional);

C - fator de uso e manejo do solo (adimensional);
P - fator de prática conservacionista do solo (adimensional).

Os fatores C e P diferenciam-se dos demais fatores por serem antrópicos, pois estão relacionados às formas de ocupação e uso das terras, não sendo dependentes apenas das condições naturais como os fatores $R, K$, L e S.

Para o cálculo da estimativa de perda de solo na Bacia Hidrográfica do Rio Una foi utilizada a ferramenta Map Algebra do ArcGIS 10.1 que efetua operações algébricas em imagens matriciais e, deste modo, possibilitou gerar o mapa de perda de solo.

\section{FATOR DE EROSIVIDADE DA CHUVA (R)}

Os dados utilizados neste estudo foram obtidos de postos pluviométricos do Sistema de Informações Hidrológicas da Agência Nacional de Águas (ANA), constituindo-se de séries históricas pluviométricas, com duração mínima de 40 anos, de 15 localidades distribuídas até $100 \mathrm{~km}$ de distância da Bacia Hidrográfica do Rio Una. Esses dados foram compilados em planilha eletrônica e calculadas as médias mensais para aplicação da fórmula proposta por Bertoni e Lombardi Neto (2012) de modo a determinar a erosividade da chuva ( $R$ ) com base nas médias mensais do Índice de Erosão (EI), como observado na Equação (2). Ainda, segundo Bertoni e Lombardi Neto (2012) esta equação estima, com relativa precisão, os fatores de erosividade para períodos longos de vinte ou mais anos.

$$
\mathrm{R}=\sum_{\mathrm{i}=1}^{12} 67,355\left(\frac{\mathrm{r}_{\mathrm{i}}^{2}}{\mathrm{P}}\right)^{0,85}
$$

Onde:

$\mathrm{R}=$ Fator de erosividade da chuva (MJ.mm.h $\mathrm{h}^{-1} \cdot \mathrm{ha}^{-}$ 1);

$r=$ Precipitação média mensal (mm);

$\mathrm{P}=$ Precipitação média anual $(\mathrm{mm})$.

Os valores de $\mathrm{R}$ obtidos para os postos pluviométricos foram posteriormente geocodificados no ArcGIS 10.1 para gerar uma malha com grade regular de $1000 \times 1000 m$ utilizando o interpolador Vizinho Natural, esta técnica não extrapola os valores, resolvendo a interpolação somente para o interior do domínio 


\section{IDENTIFICAÇÃO DE ÁREAS COM PERDA DE SOLO ACIMA DO TOLERÁVEL USANDO NDVI PARA O CÁLCULO DO FATOR C DA USLE}

dos dados. A partir dessa malha regular foram extraídos pontos com os valores de $\mathrm{R}$ para serem interpolados pelo método IDW (Inverse Distance Weighting) por assumir que cada ponto de contribuição tem influência local que diminui com a distância. Segundo Mello et al. (2003) e Gardiman Jr et al. (2012) este interpolador é amplamente utilizado com o expoente igual a dois para espacializar dados pluviais apresentando bons resultados, razão pela qual foi utilizado neste estudo.

\section{FATOR DE ERODIBILIDADE DO SOLO (K)}

O fator de erodibilidade do solo (K) foi calculado de forma indireta através da expressão de Bouyoucos (MANNIGEL et al., 2002; BERTONI; LOMBARDI NETO, 2012) que considera apenas a porcentagens das frações de areia, silte e argila, conforme a Equação (3):

$$
K=\left(\frac{\% \text { Areia }+\% \text { Silte }}{\% \text { Argila }}\right) \times \frac{1}{100}
$$

Onde:

$\mathrm{K}$ = fator erodibilidade do solo (t.ha.h/ ha.MJ.mm);

$\%$ areia, \% silte e \% argila representaram as porcentagens das respectivas frações de uma amostra de solo.

As frações de argila, silte e areia (em porcentagem) foram mensuradas pelo Instituto Agronômico de Campinas (IAC) através do método do densímetro (CAMARGO et al., 2009) para 35 pontos amostrais distribuídos regularmente na bacia hidrográfica do rio Una. Os valores de $\mathrm{K}$ obtidos para cada ponto amostral foram geocodificados no ArcGIS 10.1 e interpolados pelo método IDW (Inverse Distance Weighting). Silva et al. (2008) e Souza et al. (2010) concluíram em seus estudos que este interpolador com expoente igual a 2 apresenta resultados satisfatórios para estimar a distribuição de atributos físicos e químicos dos solos, razão pela qual foi utilizado neste estudo.

\section{FATOR TOPOGRÁFICO (LS)}

O fator topográfico foi calculado através da Equação (4) proposta por Bertoni e Lombardi Neto (2012) para a relação esperada de perdas de solo por unidade de área em um declive qualquer em relação a uma parcela unitária de 25 metros de comprimento com $9 \%$ de declive. Essa equação foi adaptada ao ambiente do ArcGIS 10.1 utilizando as ferramentas Math e Map Algebra que possibilitam efetuar operações algébricas em imagens matriciais.

$$
L S=0,00984 \times L^{0,63} \times S^{1,18}
$$

Onde:

$\mathrm{LS}=$ fator topográfico (adimensional);

$\mathrm{L}=$ comprimento de rampa (vertente) em $\mathrm{m}$;

$\mathrm{S}=$ declividade (\%).

Para determinar o fator de comprimento de rampa (L) foi empregado o algoritmo de Desmet e Govers (1996) baseado nas equações de Foster e Wischmeier (1974) expresso pela Equação (5). Esse algoritmo utiliza o conceito de contribuição de área e foi utilizado com sucesso por Silva (2004), Farinasso et al. (2006) e Freitas et al. (2007) em seus estudos para avaliar perdas de solo em Bacias Hidrográficas, razão pela qual foi utilizado nesse estudo.

$L_{i, j}=\frac{\left[\left(A_{i, j-i n}+D^{2}\right)^{m+1}-\left(A_{i, j-i n}\right)^{m+1}\right]}{\left[D^{m+2} \times x_{i, j}^{m} \times(22,13)^{m}\right]}$

Onde:

$\mathrm{Li}, \mathrm{j}$ = fator de comprimento de rampa de uma célula com coordenadas (i,j) em $m$;

$A i, j-i n=$ área de contribuição de uma célula com coordenadas $(i, j)$ em $\mathrm{m}^{2}$;

$D$ = tamanho da célula (pixel) em $m$;

$x i, j=$ valor da direção de fluxo;

$\mathrm{m}=$ coeficiente da declividade que assume os valores: 0,5 , se $s \geq 5 \%$ (s é a declividade em $\%$ ); 0,4 , se $3 \% \leq s<5 \%$; 0,3 , se $1 \% \leq s<3 \%$; e 0,2 , se $\mathrm{s}<1 \%$.

Para cada pixel calcula-se o coeficiente de declividade, a direção de fluxo e a quantidade de fluxo acumulado, facilitando o cálculo do Li,j. Assim, a partir do mapa de declividade e com o auxílio das ferramentas Math e Hydrology do ArcGIS 10.1, foi determinado o coeficiente da declividade $(m)$, a direção de fluxo que gerou o mapa de $x i, j$ e o fluxo acumulado que multiplicado pela área de cada célula $\left(1 \mathrm{~m}^{2}\right)$ gerou o mapa da área de contribuição (Ai,j-in). O 


\section{IDENTIFICAÇÃO DE ÁREAS COM PERDA DE SOLO ACIMA DO TOLERÁVEL USANDO NDVI PARA O CÁLCULO DO FATOR C DA USLE}

tamanho da célula (D), como foi definido para todas as coberturas neste trabalho, foi de $1 \mathrm{~m}$.

O mapa de Declividade (S) foi elaborado a partir da interpolação dos dados de elevação do terreno extraídos de cartas planialtimétricas do Instituto Geográfico Cartográfico (IGC) na escala 1:10.000 e por pontos cotados coletados em campo na área de estudo pelo método de interpolação TIN (Triangulated Irregular Network) que consiste em uma estrutura poliédrica cujos elementos básicos são triângulos (FELGUEIRAS, 2001).

\section{FATOR DE USO, MANEJO E PRÁtICAS CONSERVACIONISTAS DO SOLO (CP)}

Para determinar o fator $C$ foi utilizado Normalized Difference Vegetation Index (NDVI), visto a relação que há entre eles, onde o fator $C$ aumenta à medida que o NDVI diminui. Fato observado em função da diminuição da proteção oferecida pela cobertura vegetal, indicada pela diminuição no NDVI. Karaburun (2010) e Anache et al. (2014) estudaram a correlação e formulação de uma equação entre o fator C e o NDVI obtendo resultados expressivos em seus estudos, enquanto que, Carvalho et al. (2014) escalonou os valores do NDVI a partir de valores conhecidos do fator $\mathrm{C}$ obtendo resultados significativos para a distribuição do fator $\mathrm{C}$ em seus estudos, razão pela qual foi utilizado nesse estudo.
O NDVI foi gerado a partir da banda espectral do infravermelho próximo (IP) e a banda do vermelho (V) do satélite SPOT5 como mostrado na equação (6):

$$
N D V I=\frac{(I P-V)}{(I P+V)}
$$

Após gerar a imagem do NDVI, utilizouse o mapa de uso do solo e cobertura vegetal, que foi gerado a partir da imagem do satélite SPOT5 pelo método da classificação supervisionada multivariada de Máxima Verossimilhança do software ArcGIS 10.1, para identificar e segmentar os usos de interesses na Bacia Hidrográfica do Rio Una. Os usos de interesse para obter os valores do fator $C$ para utilização da imagem do NDVI foram: Agricultura com Culturas Perenes e Culturas Temporárias, Pastagens, Campos Sujos, Área Florestal e Edificações Antrópicas Presentes em Áreas Rurais, este último foi considerado, pois não há uma impermeabilização do solo nessas áreas. A partir desses usos do solo foi gerado um plano de informação (PI) para a extração dos valores do NDVI dessas áreas.

As escalas dos valores de NDVI e do fator C são inversas e não têm a mesma proporcionalidade, pois o NDVI varia de -1 a 1 e o fator $C$ de 0 a 1 (Tabela 1). Para o NDVI, as áreas com mais cobertura vegetal apresentam valores tendendo a 1 , enquanto que, para o fator $C$ os valores mais próximos de 1 se referem aos locais menos vegetados.

Tabela 01 - Fator C para alguns usos do solo

\begin{tabular}{|c|c|}
\hline Usos do Solo & Fator C \\
\hline Mata e vegetação nativa (3) & 0,0004 \\
\hline Reflorestamento de eucalipto (3) & 0,0470 \\
\hline Agricultura de sequeiro ou irrigada (2) & 0,1800 \\
\hline Pastagem degradada (1) & 0,0500 \\
\hline Pastagem Nativa (1) & 0,0050 \\
\hline Área Urbana (1) & 0,0000 \\
\hline Corpos de água (lagos) (1) & 0,0000 \\
\hline Cursos de água (rios) (1) & 0,0000 \\
\hline Solo exposto (arado e ou gradeado desprovido de cobertura vegetal) (1) & 1,0000 \\
\hline
\end{tabular}

Fonte: (1)Stein et al. (1987), (2)Silva (2004), (3)Silva et al. (2010). 


\section{IDENTIFICAÇÃO DE ÁREAS COM PERDA DE SOLO ACIMA DO TOLERÁVEL USANDO NDVI PARA O CÁLCULO DO FATOR C DA USLE}

Dessa forma, foi proposta uma nova equação (7) para que os valores de NDVI ficassem com a mesma proporcionalidade do fator $\mathrm{C}$ baseando-se nos valores de mata e agricultura presentes na Tabela 1, uma vez que, foram utilizados os valores médios do NDVI de áreas com uso do solo conhecidos de 0,8974 e - 0,6923 correspondentes às áreas de mata e agricultura, respectivamente, identificados na área de estudo.

$$
N D V I_{\text {esc }}=-0,1130 \times N D V I+0,1018
$$

Onde:

NDVlesc é o valor escalonado do NDVI

Para os usos do solo classificados como edificações urbanas e lagos foi criado um Plano de Informação (PI) a parte e atribuído o valor igual a zero, enquanto que, para o restante do PI foi atribuído o valor igual a 1. Posteriormente, foi utilizada a ferramenta Map Algebra do ArcGIS 10.1 para multiplicar o PI que contêm os valores de NDVI escalonados pelo PI que contêm os valores do uso do solo para edificações urbanas e lagos gerando assim o mapa do fator $\mathrm{C}$.

$O$ fator $(P)$ é a relação entre a intensidade de perdas de terra com determinada prática conservacionista e aquelas quando a cultura está plantada no sentido do declive (morro abaixo). Para o fator $\mathrm{P}$ foi atribuído o valor 1 , adotando-se a metodologia proposta por Lee (2004) e Aziz et al. (2012), que recomenda que se não existem práticas conservacionistas no solo, deve ser atribuído o valor 1 ao fator $\mathrm{P}$ e ainda ressaltam que os efeitos dos dois fatores (C e P) podem ser avaliados conjuntamente devido à estreita relação entre ambos.

Assim os fatores C e P foram analisados como um único fator neste estudo, uma vez que, durante os trabalhos de campo não foi encontrado o uso de práticas conservacionista ao longo da bacia hidrográfica.

\section{IDENTIFICAÇÃO DAS ÁREAS COM PERDA DE SOLOS ACIMA DA FAIXA TOLERÁVEL}

O mapa da distribuição de perda de solos foi reclassificado de acordo com a tolerância de perda de solo para o Estado de São Paulo calculada por Mannigel et al. (2002). Para isso, utilizou-se o mapa pedológico do Estado de São Paulo produzido pelo IAC na escala de 1:500.000, onde o mapa foi vetorizado nos limites da Bacia Hidrográfica do Rio Una, sendo identificado três classes de solos: o PVA18 (Argissolos Vermelhos-Amarelos Distróficos A moderado textura média/argilosa e argilosa relevo ondulado e forte ondulado); o LVA1(Latossolos Vermelhos-Amarelos Distróficos A moderado textura argilosa relevo forte ondulado) e o LVA17 (Latossolos VermelhosAmarelos Distróficos relevo ondulado + Cambissolos Háplicos distróficos relevo ondulado e forte ondulado ambos A moderado textura argilosa).

Os valores verificados de tolerância de perda de solo para o PVA18, LVA1 e LVA17 foram de, respectivamente, 9,06t/ha.ano, $11,53 t /$ ha.ano e $13,12 t /$ ha.ano, sendo que o valor do LVA17 é resultado de uma média entre os valores do Latossolos Vermelhos-Amarelos Distróficos A moderado textura argilosa (11,53t/ha.ano) e Cambissolos Háplicos Distróficos A moderado textura argilosa (14,70t/ha.ano).

A partir do mapa pedológico e do mapa de distribuição de perda de solo foi realizado uma operação algébrica de subtração dos mapas utilizando a ferramenta Map Algebra do ArcGIS 10.1. O mapa de tolerância de perda de solo foi subtraído do mapa de perda de solo, sendo que os valores negativos ou iguais a zero indicam que a perda de solo é tolerável, enquanto que, os valores positivos e maiores que zero indicam que a perda de solo está acima da tolerável. Para a classificação da perda de solo como tolerável atribuiu-se o valor igual a 5 e para a classificação da perda de solo acima do tolerável foi atribuído o valor igual a 1 .

\section{RESUTADOS}

Os resultados da análise da erosividade das chuvas ( $R$ ) na área de estudo (Figura 2A) mostram que o valor médio anual encontrado para a área de estudo foi de 6660 MJ.mm.ha${ }^{1}$.ano ${ }^{-1}$, sendo que a região sul da bacia apresenta maior intensidade de chuvas e por consequência 


\section{IDENTIFICAÇÃO DE ÁREAS COM PERDA DE SOLO ACIMA DO TOLERÁVEL USANDO NDVI PARA O \\ CÁLCULO DO FATOR C DA USLE}

tem o valor de erosividade maior. De forma geral, a área em estudo apresentou baixa variabilidade espacial da erosividade das chuvas, variando de 6430.38 a $6953.40 \mathrm{MJ} . \mathrm{mm} \cdot$ ha $^{-1}$. ano-1 (Figura 2A).

Essa constância da erosividade deve-se ao tamanho da área estudada $\left(96,4 \mathrm{Km}^{2}\right)$, tornando possível maior uniformidade da distribuição da chuva sobre a mesma.

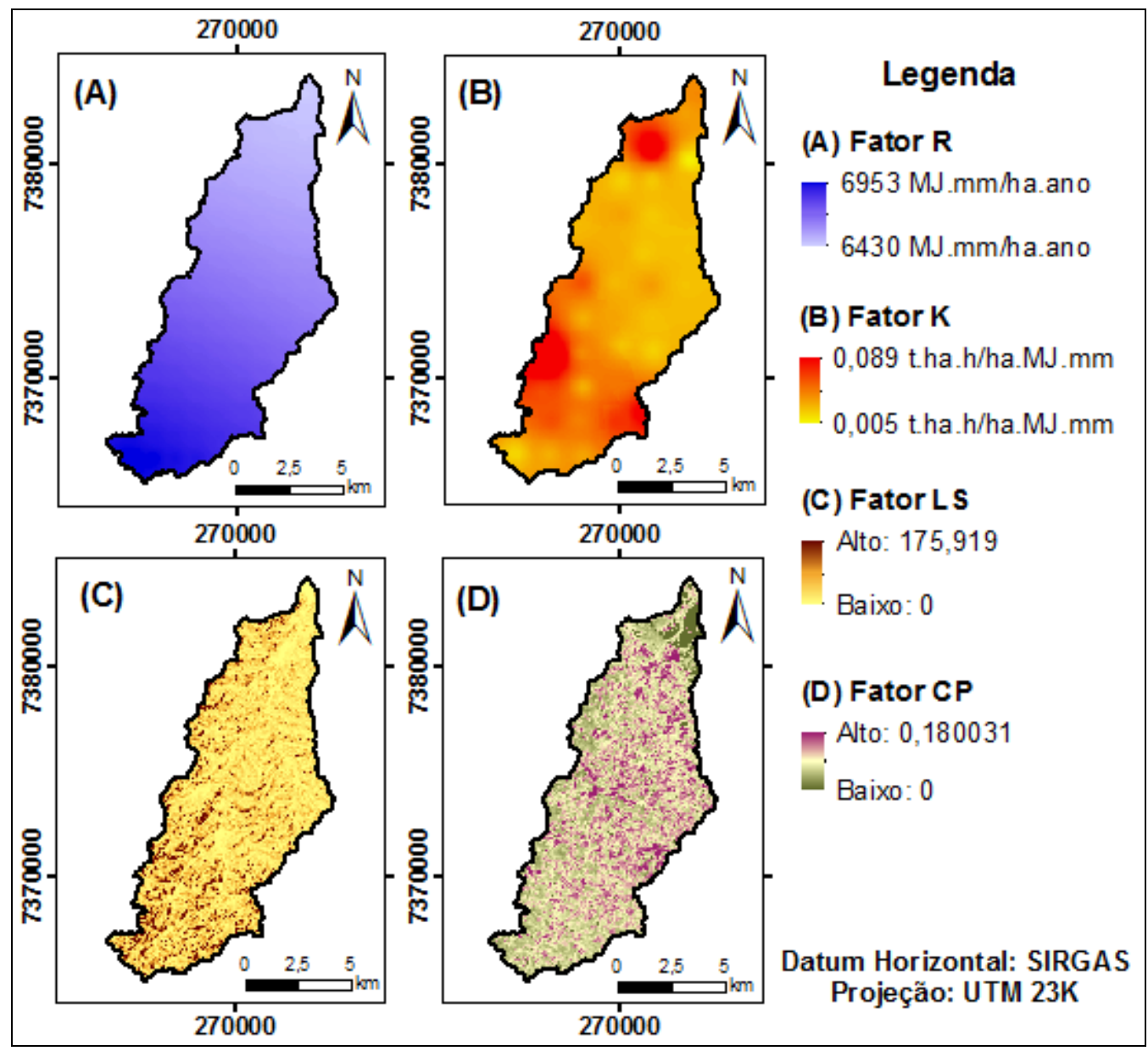

Figura 02 - Mapas dos fatores utilizados na USLE

Os valores de erodibilidade do solo (Fator K) obtidos pela Equação (3) de acordo com os valores de granulometria amostrados em campo, a região sul da bacia hidrográfica (Figura 2B) apresenta os maiores valores, entretanto, é a área mais preservada da bacia, o que ameniza a perda de solo devido a presença de cobertura vegetal (matas). Em uma pequena parte da região norte da bacia hidrográfica há presença de altos valores de erodibilidade e como na região sul da bacia há também a presença de cobertura vegetal amenizando a perda de solo.

O valor médio de erodibilidade do solo para este estudo foi de 0,02 t.ha.h/ha.MJ.mm, apresentando grande variabilidade espacial em sua distribuição na bacia com valor mínimo de aproximadamente 0,004 t.ha.h/ha.MJ.mm e valor máximo de aproximadamente 0,089 t.ha.h/ha.MJ.mm, os quais estão dentro da faixa de valores extremos aceitáveis para o Estado de São Paulo (MANNIGEL et al., 2002). 


\section{IDENTIFICAÇÃO DE ÁREAS COM PERDA DE SOLO ACIMA DO TOLERÁVEL USANDO NDVI PARA O \\ CÁLCULO DO FATOR C DA USLE}

O Fator LS (Figura 2C) representa a relação entre a declividade (em \%) pelo comprimento de rampa (em metros) que potencializa a força erosiva da água (SILVA, 2004; BERTONI; LOMBARDI NETO, 2012).

$\mathrm{Na}$ Figura $1 \mathrm{C}$ é possível identificar as áreas onde o Fator LS tem maior influência, como na porção sul da bacia, que apresenta as maiores altitudes e declividades. As áreas com os menores valores para o Fator Topográfico é a região norte, onde se localiza a área urbana e as menores altitudes e declividades da bacia.

Os maiores valores encontrados do fator CP (Figura 2D) são encontrados no médio Rio Una, isto é, na região central da bacia hidrográfica onde a prática da agricultura é mais intensa. Na região norte há presença de valores nulos devido a presença de áreas urbanizadas, uma vez que, essas áreas geralmente apresentam grandes percentuais de áreas impermeabilizadas, e desta maneira, não expõe o solo aos processos erosivos.

Há outras áreas com valores do fator $\mathrm{CP}$ nulo espalhadas por toda bacia que correspondem aos corpos de água, sendo que, juntas, as áreas urbanizadas e os corpos de água correspondem a aproximadamente $3,56 \%$ da área total da bacia.

$\mathrm{O}$ valor médio do fator $\mathrm{CP}$ para a área de estudo foi 0,079 , isto é, baixo se comparado ao fator C obtido por outros estudos em bacias hidrográficas como os de Karaburun (2010) que variou de 0,2 a 0,4 ou de Anache et al. (2014) que ficou acima de 0,23 para todos os modelos testados.

As perdas de solo estimadas para $36,92 \%$ da área de estudo apresentam valor menor que 1 t.ha-1.ano-1 e correspondem às áreas mais planas e/ou urbanizadas da bacia hidrográfica (Figura 3), enquanto que, mais de 93,4\% da área de estudo apresentou perdas de solos abaixo de 10 t.ha ${ }^{-1}$.ano ${ }^{-1}$ podendo ser classificada, segundo FAO (1980), como de baixa susceptibilidade à erosão, ou seja, apresenta nenhuma ou ligeira perda de solo. 


\section{IDENTIFICAÇÃO DE ÁREAS COM PERDA DE SOLO ACIMA DO TOLERÁVEL USANDO NDVI PARA O CÁLCULO DO FATOR C DA USLE}

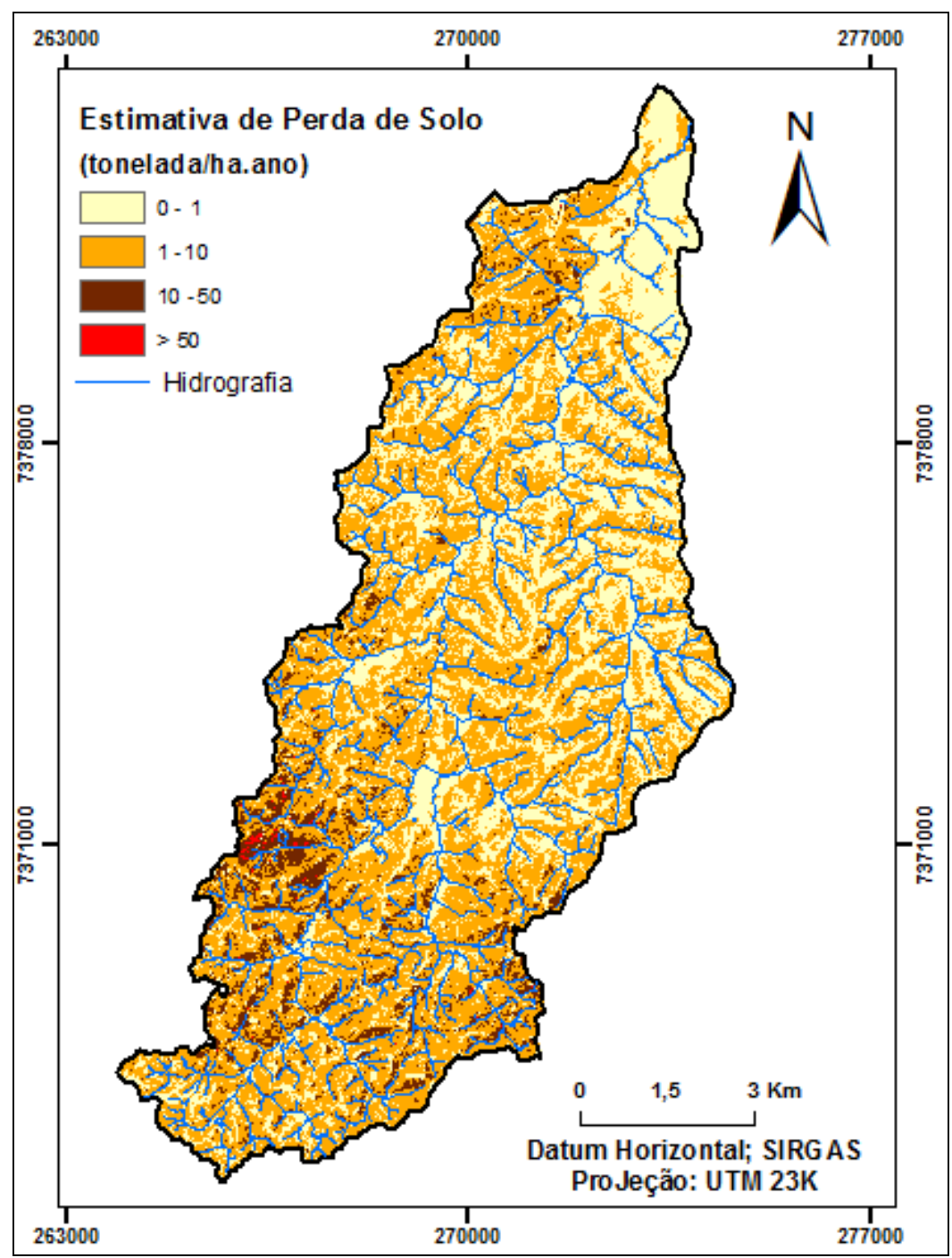

Figura 03 - Mapa da estimativa de perdas anuais de solo por erosão da área de estudo

Observa-se ainda que apenas $0,17 \%$ da área apresentou perda de solos acima 50 t.ha${ }^{1}$.ano ${ }^{-1}$ e, segundo FAO (1980), estas áreas se enquadram na classe de alto grau de susceptibilidade à erosão. As áreas com perda de solos acima de 50 t.ha ${ }^{-1}$. ano ${ }^{-1}$ encontram-se em regiões com declividades média de aproximadamente $67,35 \%$, ou seja, regiões com altas declividades e, portanto, a declividade, neste caso, é o principal fator atuante na perda de solos na Bacia Hidrográfica do Rio Una.

Essa bacia hidrográfica pode ser classificada como de baixa susceptibilidade à erosão ou perda de solo, uma vez que, a perda de solo média estimada para a região é de aproximadamente 3,22 t.ha ${ }^{-1}$ ano $^{-1}$. Estes resultados evidenciam a importância das áreas que apresentam cobertura florestal preservada, visto que essa bacia hidrográfica tem aproximadamente $41 \%$ de sua extensão territorial dominada por áreas florestais.

As áreas que apresentaram alta perda de solo são dominadas por declividades acentuadas com presença de atividades agrícolas, o que evidencia a adoção de práticas conservacionistas para minimizar a degradação do solo.

A Figura 4 mostra o mapa pedológico $(A)$ e o mapa de perda de solo classificado (B) da Bacia Hidrográfica do Rio Una, enquanto que, a Figura 5 mostra o mapa de uso do solo e cobertura vegetal. 


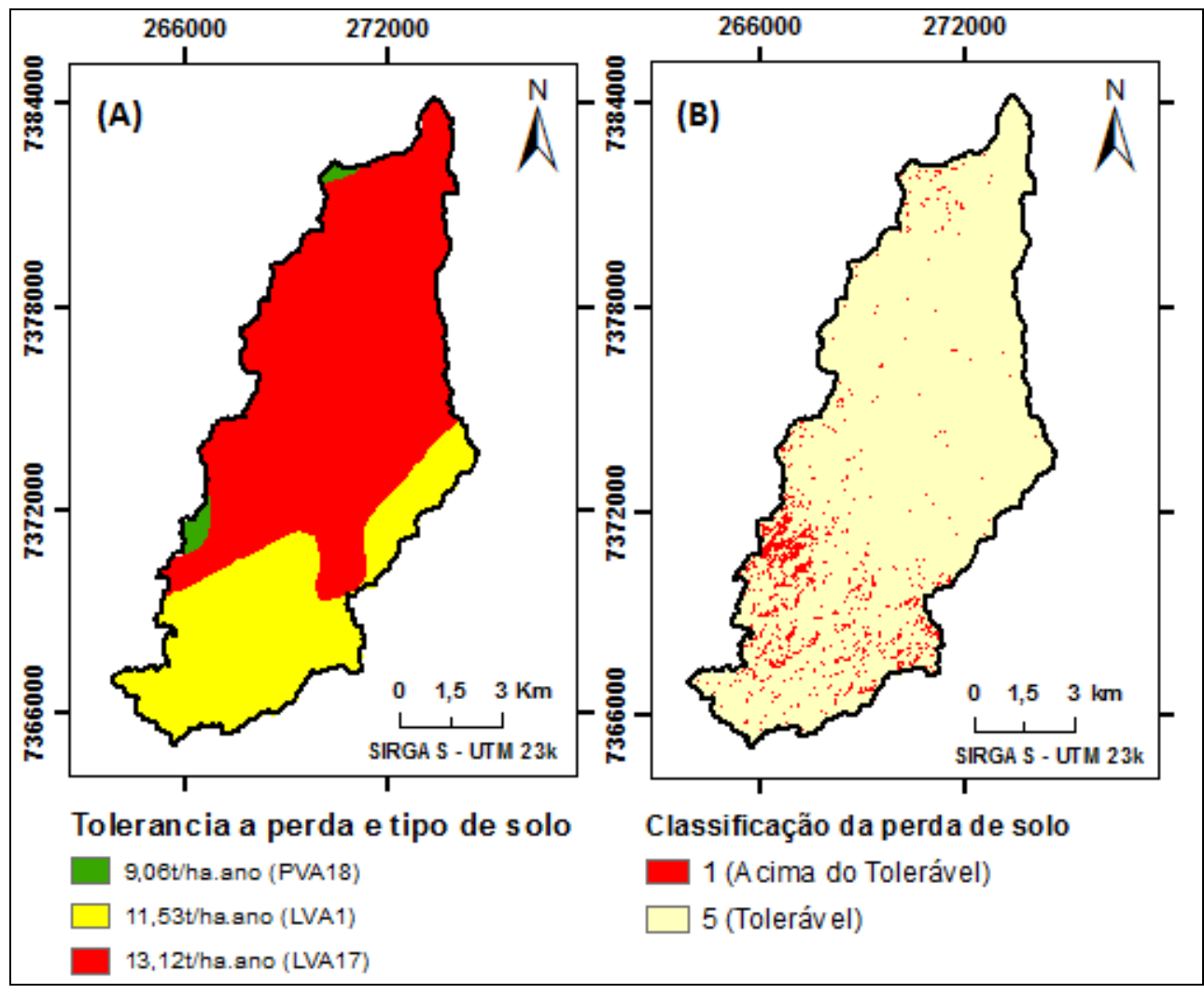

Figura 04 - Mapa pedológico (A) e de perda de solo classificado (B)

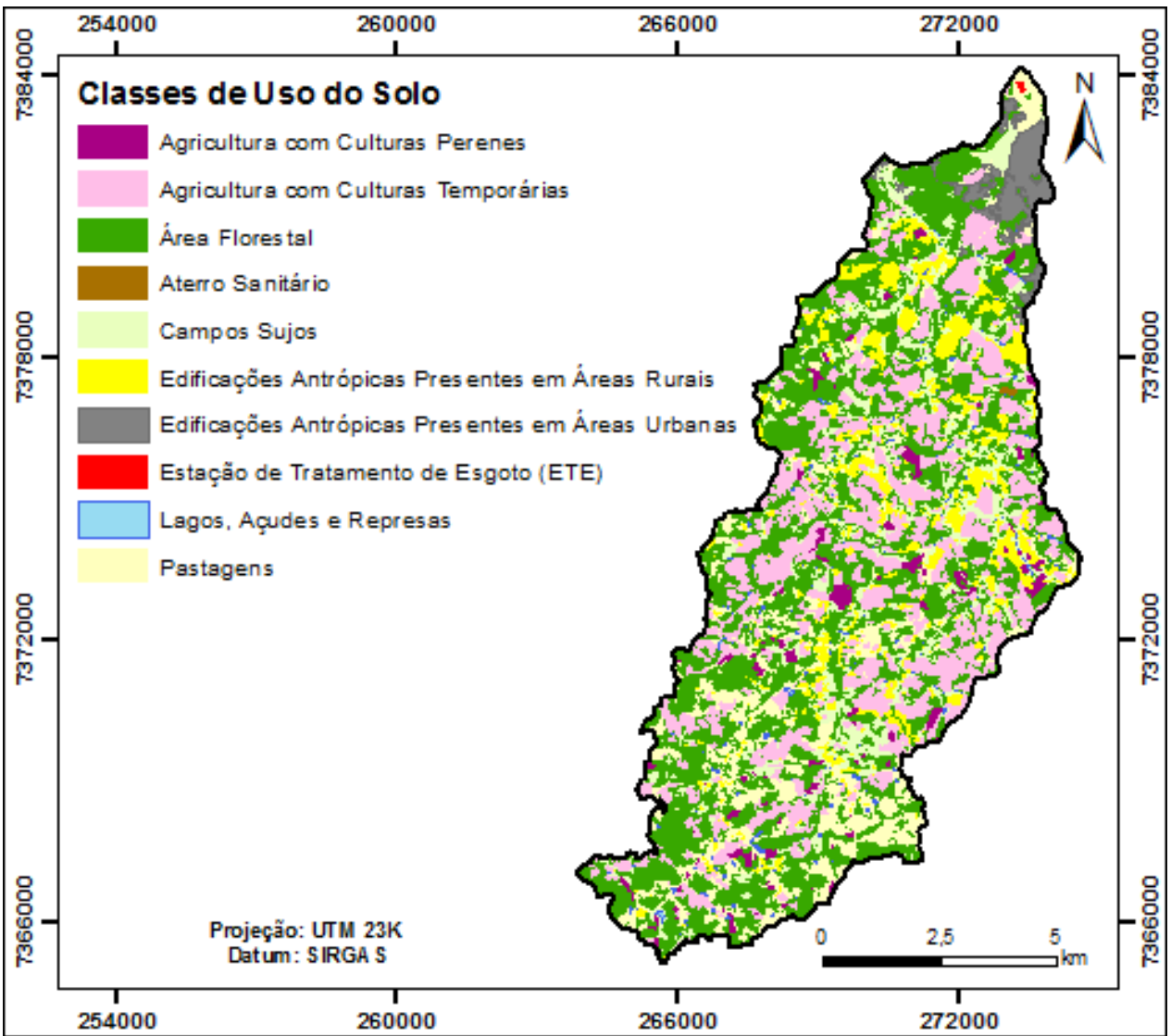

Figura 05 - Mapa de uso do solo e cobertura vegetal 


\section{IDENTIFICAÇÃO DE ÁREAS COM PERDA DE SOLO ACIMA DO TOLERÁVEL USANDO NDVI PARA O CÁLCULO DO FATOR C DA USLE}

Aproximadamente $95,2 \%$ da área do mapa de perda de solo classificado encontra-se na faixa de perda de solo tolerável, enquanto que, aproximadamente $4,8 \%$ encontra-se na faixa de perda acima do tolerável (Figura 4B). Em relação ao mapa pedológico, a Bacia Hidrográfica tem aproximadamente $1,25 \%$ de sua área classificada como PVA18, 32,7\% como LVA1 e 66,05\% como LVA17 (Figura 4A).

Comparando-se o mapa da Figura 4B com o mapa da Figura 5 de uso do solo e cobertura vegetal, percebe-se que a maior parte das áreas identificadas com perda de solo acima do tolerável encontram-se em áreas com culturas agrícolas e relevo extremamente acidentados, demonstrando que as atividades antrópicas nessas áreas provocam um desequilíbrio, interferindo no regime natural de perda de solos por erosão hídrica provenientes de eventos pluviométricos.

\section{CONCLUSÃO}

A utilização do ArcGIS 10.1 para a aplicação da USLE e obtenção do fator $C$ a partir da utilizado do NDVI para tal demonstrou-se bastante eficaz, pois apresentou coerência com dados obtidos por outros autores que utilizaram outros softwares em ambiente de Sistemas de Informações Geográficas (SIG's) e métodos diretos (SILVA, 2004; FARINASSO et al., 2006; FREITAS et al., 2007). Além disso, o NDVI permitiu avaliar dentre as culturas agrícolas, aquelas que apresentam uma maior exposição do solo, portanto, permitindo a obtenção de um valor para o fator $\mathrm{C}$ menos subjetivo.

O grande percentual de áreas cobertas por matas foi responsável por manter as taxas de erosão menores que 10 t.ha-1 ano $^{-1}$ em 93,4\% da área de estudo, enquanto que, as áreas cobertas por matas e área urbana foi o responsável por manter os valores do fator $C$ baixos para grande parte da área de estudo. As áreas que apresentaram taxas de erosão superiores a 10 t.ha ${ }^{-1}$.ano $^{-1}$ encontram-se em locais com atividades agrícolas e com altas declividades.

Esse método pode ser utilizado para tomada de decisão por órgãos públicos ou por interessados na preservação do solo, pois é de fácil aplicabilidade e entendimento, podendo ser utilizado em diferentes áreas como um subsídio no manejo do solo visando a preservação do mesmo.

\section{REFERÊNCIAS}

AMORIM, R. S. S.; SILVA, D. D.; PRUSKI, F. F.; MATOS, A. T. Avaliação do desempenho dos modelos de predição da erosão hídrica USLE, RUSLE e WEPP para diferentes condições edafoclimáticas do Brasil. Revista de Engenharia Agrícola, v.30, n.6, p. 1046-1049, 2010.

ANACHE, J. A. A.; BACCHI, C. G.; ALVESSOBRINHO, T. Modeling of (R) USLE C-factor for pasture as a function of Normalized Difference Vegetation Index. European International Journal of Science and Technology, v. 3, n. 9, 2014.

AZIZ, S. A.; STEWARD, B. L.; KALEITA, A. L.; KARKEE, $M$. Assessing the effects of DEM uncertainty on erosion rate estimation in an agricultural field. Transactions of the ASABE, v. 55, n. 3, p. 785, 2012.

BERTONI, J.; LOMBARDI NETO, F. Conservação do Solo. - 8a. ed. - São Paulo: Ícone, 2012.

BERTONI, J.; LOMBARDI NETO, F. Conservação do solo. Livroceres, Piracicaba - SP, 1999. 355 p.

CAMARGO, O. A.; MONIZ, A. C.; JORGE, J. A.; VALADARES, J. M. A. S. Métodos de Analise Química, Mineralógica e Física de Solos do Instituto Agronômico de Campinas. Campinas, Instituto Agronômico/Fundação IAC, 2009. 77 p. (Boletim técnico, 106, Edição revista e atualizada) CARVALHO, D. F.; DURIGON, V. F.; ANTUNES, M. A. H.; ALMEIDA, W. S.; OliveiRA, P. T. S. Predicting soil erosion using Rusle and NDVI time series from TM Landsat 5. Pesquisa agropecuária brasileira, Brasília, v. 49, n. 3, 2014.

costA, A. L. C. Estudo da vulnerabilidade à erosão com a aplicação da Equação Universal de Perda do Solo na Alta Bacia Hidrográfica do Rio Jacaré Pepira, utilizando SIG/SPRING. Dissertação (mestrado), Universidade Estadual Paulista, IGCE, Rio Claro, 2005.

DESMET, P. J. J.; GOVERS, G. A GIS procedure for automatically calculating the USLE LS factor on topographically complex landscape units. Journal 


\section{IDENTIFICAÇÃO DE ÁREAS COM PERDA DE SOLO ACIMA DO TOLERÁVEL USANDO NDVI PARA O CÁLCULO DO FATOR C DA USLE}

of Soil and Water Conservation, v. 51, n.5, p. 427433, 1996.

FAO - FOOD AND AGRICULTURE ORGANIZATION. La erosión del suelo por el agua: Algunas medidas para combatirla en las tierras de cultivo. Cuadernos de fomento agropecuário de la Organización de Las Naciones Unidas, Roma: FAO, n.81, 1980, 207p.

FARINASSO, M.; CARVALHO JÚNIOR, O. A. D.; GUIMARÃES, R. F.; GOMES, R. A. T.; RAMOS, V. M. Avaliação Qualitativa do Potencial de Erosão Laminar em Grandes Áreas por Meio da EUPS Equação Universal de Perdas de Solos Utilizando Novas Metodologias em SIG para os Cálculos dos seus Fatores na Região do Alto Parnaíba PI-MA. Revista Brasileira de Geomorfologia, v. 7, n. 2, 2006.

FELGUEIRAS, C. A. Modelagem numérica de terreno. In: CÂMARA, G.; DAVIS, C. e MONTEIRO, A. M. V. Introdução à Ciência da Geoinformação. São José dos Campos: Instituto Nacional de Pesquisas Espaciais (INPE), 2001.

FOSTER, G. R.; WISCHMEIER, W. H. Evaluating irregular slopes for soil loss prediction. Trans ASAE Gen Ed Am Soc Agric Eng, v.17, p. 305-309, 1974.

FREITAS, L. F. D.; CARVALHO JÚNIOR, O. A. D.; GUIMARÃES, R. F.; GOMES, R. A. T.; MARTINS, É. D. S.; GOMES-LOEBMANN, D. Determinação do potencial de erosão a partir da utilização da EUPS na Bacia do Rio Preto. Revista Espaço e Geografia, v. 10, n. 2, p. 431-452, 2007.

FUGIHARA, A. K. Predição de Erosão e Capacidade de Uso do Solo numa microbacia do Oeste Paulista com suporte de Geoprocessamento. Dissertação (Mestrado) Escola Superior de Agricultura "Luiz de Queiroz", Piracicaba, 2002. p. 118.

GALDINO, S.; WEILL, M. A. M. Estimativa do fator topográfico (LS) da RUSLE para a bacia do alto Taquari - MS/MT, utilizando algoritmo do ângulo de inclinação limite para erosão/deposição. In Anais do XV Simpósio Brasileiro de Sensoriamento Remoto - SBSR, Curitiba, Brasil. INPE, 2011, pp. 4813.

GARDIMAN Jr, B. S.; MAGALHÃES, I. A. L.; FREITAS, C. A. A.; CECÍlIO, R. A. Análise de técnicas de interpolação para espacialização da precipitação pluvial na bacia do rio Itapemirim (ES). Ambiência, v. 8, n. 1, p. 61-71, 2012.

IBGE - Instituto Brasileiro de Geografia e Estatística. 1979. Biblioteca. Rio de Janeiro: IBGE. Disponível em: <http://biblioteca.ibge.gov.br/>. Acesso em: 13 de outubro 2015.

IBGE - Instituto Brasileiro de Geografia e Estatística. Base Estatcart de Informações do Censo Demográfico 2010: Resultados do Universo por Setor Censitário. Rio de Janeiro: IBGE, 2012. CD-ROM.

IBGE - Instituto Brasileiro de Geografia e Estatística. Manual Técnico de Uso da Terra. 3 ed. Rio de Janeiro: IBGE, 2013. 171 p.

KARABURUN, A. Estimation of C factor for soil erosion modeling using NDVI in Buyukcekmece watershed. Ozean journal of applied sciences, v. 3, n. 1, p. 77-85, 2010.

LEE, S. Soil erosion assessment and its verification using the Universal Soil Loss Equation and Geographic Information System: a case study at Boun, Korea. Environmental Geology, 45(4): 457465, 2004.

MANNIGEL, A. R.; PASSOS, M.; MORETI, D.; ROSA MEDEIROS, L. Fator erodibilidade e tolerância de perda dos solos do Estado de São Paulo. Acta Scientiarum. Agronomy, v. 24, p. 1335-1340, 2002.

MEDEIROS, P.H.A.; GÜNTNER, A.; FRANCKE, T.; MAMEDE, G.L.; de ARAÚJO, J.C. Modelling spatio-temporal patterns of sediment yield and connectivity in a semi-arid catchment with the WASA-SED model. Hydrological Sciences Journal, v.55, n.4, 2010, pg. 636-648.

MELLO, C. R.; LIMA, J. M.; SILVA, A. M.; MELLO, J. M.; SILVA, M. S. Krigagem e inverso do quadrado da distância para interpolação dos parâmetros da equação de chuvas intensas. Revista Brasileira de Ciência do Solo, Viçosa, v.27, n.5, p.925-933, 2003.

MINGOTI, R. Produção de sedimentos em microbacias hidrográficas em função do relevo e da cobertura florestal. Dissertação (mestrado), Escola Superior de Agricultura "Luiz de Queiroz", Piracicaba, 2009. 101p. 


\section{IDENTIFICAÇÃO DE ÁREAS COM PERDA DE SOLO ACIMA DO TOLERÁVEL USANDO NDVI PARA O \\ CÁLCULO DO FATOR C DA USLE}

RENARD, K. G.; FOSTER, G. R.; WEEIES, G. A.; MCCOOL, D. K.; YODER, D. C. Predicting soil erosion by water: a guide to conservation planning with the revised universal soil loss equation (RUSLE). Agriculture Handbook (Washington), n.703, 1997.

Sales, J. C. A., 2015. Metodologia para identificação de áreas de risco e prioritárias para conservação da avifauna na Bacia Hidrográfica do rio Una, Ibiúna/SP. 2015. 131 f. Dissertação (mestrado) - Universidade Estadual Paulista Júlio de Mesquita Filho, Campus Experimental de Sorocaba.

Salles, M. H. D.; Conceição, F. T.; Angelucci, V. A.; Sia, R.; Pedrazzi, F. J. M.; Carra, T. A.; Navarro, G. R. B., 2008. Avaliação Simplificada de Impactos Ambientais na Bacia do Alto Sorocaba (SP). Revista de estudos ambientais. v.10, n. 1, p. 6-20. SEADE - Fundação Sistema Estadual de Análise de Dados. 2015. Informação dos Municípios Paulistas (IMP). Disponível em: < http://www.imp.seade.gov.br/>. Acesso em: 14 Out. 2015.

SILVA, F. D. G.; MINOTTI, F.; LOMBARDI NETO, F.; PRIMAVESI, O.; CRESTANA, S. Previsão da perda de solo na Fazenda Canchim-SP (EMBRAPA) utilizando geoprocessamento e o USLE 2D. Eng Sanit Ambient, v. 15, n. 2, p. 141-148, 2010.

SILVA, S. D. A.; SOUZA LIMA, J. S. D.; SOUZA, G. S. D.; OLIVEIRA, R. B. D. Avaliação de interpoladores estatísticos e determinísticos na estimativa de atributos do solo em agricultura de precisão. Idesia (Arica), v. 26, n. 2, p. 75-81, 2008.

SILVA, V. C. Estimativa da erosão atual da bacia do Rio Paracatu (MG/GO/DF). Pesquisa Agropecuária Tropical (Agricultural Research in the Tropics), v. 34, n. 3, p. 147-159, 2004.

SOUZA, G. D.; LIMA, J. D. S.; XAVIER, A. C.; ROCHA, W. D. Krigagem ordinária e inverso do quadrado da distância aplicados na espacialização de atributos químicos de um argissolo. Scientia Agraria, v. 11, n. 1, p. 073-081, 2010.

STEIN, D. P.; DONZELLI, P. L.; GIMENEZ, A. F.; PONÇANO, W. L.; LOMBARDI NETO, F. Potencial de erosão laminar, natural e antrópica, na Bacia do Peixe Paranapanema. Anais. Simpósio
Nacional de Controle de Erosão, v. 4, p. 105-135, 1987.

Veloso, H. P., Rangel Filho, A. L. R. Lima, J. C. A., 1991. Classificação da Vegetação Brasileira Adaptada a um Sistema Universal. Ministério da Economia, Fazenda e Planejamento, Fundação Instituto Brasileiro de Geografia e Estatística, Diretoria de Geociências, Departamento de Recursos Naturais e Estudos Ambientais.

WISCHMEIER, W.H.; SMITH, D.D. Predicting rainfall erosion losses - a guide to conservation planning. Washington, USDA, Agricultural Handbook, (537), 1978. 58p 